\title{
Dose response of sodium glucose cotransporter-2 inhibitors in relation to urinary tract infections: a systematic review and network meta-analysis of randomized controlled trials
}

\author{
Jennifer R. Donnan BScPharm MSc, Catherine A. Grandy, Eugene Chibrikov PhD, \\ Carlo Marra PharmD PhD, Kris Aubrey-Bassler MD, Karissa Johnston PhD, Michelle Swab MLIS, \\ Jenna Hache BScPharm, Daniel Curnew BScPharm, Hai Nguyen PhD, \\ John-Michael Gamble BScPharm PhD
}

\section{Abstract}

Background: The sodium glucose cotransporter-2 (SGLT2) inhibitors are a novel group of drugs for treatment of type 2 diabetes mellitus. We investigated whether there is a dose-response relation between SGLT2 inhibitors and urinary tract infections (UTIs) in patients with type 2 diabetes, relative to other diabetes therapies or placebo.

Methods: We conducted a systematic review and network meta-analysis of randomized controlled trials (RCTs) of SGLT2 inhibitors in patients with type 2 diabetes. We searched 6 databases and the reference lists of key papers. We included studies with placebo or active antidiabetic comparators that reported the outcome of UTI, and established thresholds for high and low doses of SGLT2 inhibitors. We used a random-effects model to estimate the pooled effect estimates and $95 \%$ credible intervals.

Results: We screened 2418 citations and included 105 references for studies of 8 unique SGLT2 inhibitors, representing 60082 individuals (with a total of 4348 UTIs). Most mixed-treatment comparisons showed no significant difference in risk of UTI, with the exception of high-dose dapagliflozin ( $\geq 10 \mathrm{mg}$ ) compared with placebo (odds ratio [OR] 1.30, 95\% credible interval 1.09-1.57), with active comparators (OR 1.44, 95\% credible interval 1.15-1.79), with empagliflozin at both low (OR 1.30, 95\% credible interval 1.04-1.60) and high (OR 1.39, 95\% credible interval 1.12-1.72) doses, and with low-dose ertugliflozin (OR 1.43, 95\% credible interval 1.012.01). When the analysis was restricted to RCTs with a low risk of bias, the results were nonsignificant.

Interpretation: Current RCT evidence does not suggest a dose-response relation between most SGLT2 inhibitors and UTIs, with the exception of dapagliflozin. Further research is needed to quantify the relation between SGLT2 inhibitors and more serious infections. Trial registration: PROSPERO registration no. CRD42016038715.

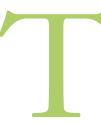
he sodium glucose cotransporter-2 (SGLT2) inhibitors are a novel group of drugs for the treatment of type 2 diabetes mellitus. These products have several benefits, including a moderate glycemic-lowering effect, low risk of hypoglycemia, reductions in weight and blood pressure, and reduction in major adverse cardiovascular events. ${ }^{1,2}$ The SGLT2 inhibitors are recommended as one of several options for second-line therapy, with empagliflozin and canagliflozin specifically recommended in clinical guidelines as the preferred second-line therapies for patients with pre-existing cardiovascular disease. ${ }^{3}$ Their unique mechanism of action - inhibition of the reabsorption of glucose at the proximal renal tubule - results in increased urinary glucose excretion ${ }^{4}$ and has led to speculation about an increased risk of urinary tract infections (UTIs). ${ }^{5}$ According to a public safety advisory in the United States, there were 19 reported cases of life-threatening kidney or blood infection between
March 2013 and October 2014 that originated as a UTI in individuals taking SGLT2 inhibitors. ${ }^{6}$

Although product monographs for SGLT2 inhibitors identify the increased risk of UTI as a potential adverse effect, clinical trial evidence to date does not support this notion. Two published meta-analyses of randomized controlled trials (RCTs) found no increased risk of UTIs, ${ }^{7,8}$ except within a subgroup of dapagliflozin users receiving a 10 -mg dose,${ }^{8}$ which indicated a potential dose-response

Competing interests: None declared.

This article has been peer reviewed.

Correspondence to: John-Michael Gamble,jm.gamble@ uwaterloo.ca CMAJ Open 2018. DOI:10.9778/cmajo.20180111 
relation. Such a dose-response relation is plausible, given variation in the rate of urinary glucose excretion with individual agents (Table 1). Some of these agents have shown a clear dose-response relation, whereas others seem to reach a maximum for urinary glucose excretion with certain dosages. Moreover, prior meta-analyses were limited to studies with at least 24 weeks of follow-up. It is unlikely that development of a UTI would require months of treatment, and therefore data from short-term studies should also be considered.

The specific question that we addressed in this systematic review and network meta-analysis was whether there is a

\begin{tabular}{|c|c|c|}
\hline Study & Dose (mean 24-h urinary glucose excretion) & Dose response \\
\hline \multicolumn{3}{|l|}{ Empagliflozin } \\
\hline Zhao et al. ${ }^{9}$ & $\begin{array}{l}\text { Day 1: } 10 \mathrm{mg}(88 \mathrm{~g}), 25 \mathrm{mg}(83 \mathrm{~g}) \\
\text { Day 9: } 10 \mathrm{mg}(96 \mathrm{~g}), 25 \mathrm{mg}(83 \mathrm{~g})\end{array}$ & $\begin{array}{l}\text { No difference between } 10-\mathrm{mg} \text { and } \\
25 \text {-mg doses }\end{array}$ \\
\hline Kanada et al. ${ }^{10}$ & $\begin{array}{l}\text { Day 1: } 1 \mathrm{mg}(40 \mathrm{~g}), 5 \mathrm{mg}(80 \mathrm{~g}), 10 \mathrm{mg}(85 \mathrm{~g}), 25 \mathrm{mg} \mathrm{(90} \mathrm{g)} \\
\text { Day 27: } 1 \mathrm{mg}(41 \mathrm{~g}), 5 \mathrm{mg}(77 \mathrm{~g}), 10 \mathrm{mg}(81 \mathrm{~g}), 25 \mathrm{mg}(93 \mathrm{~g}) \\
\text { (estimated from chart) }\end{array}$ & Dose response \\
\hline Scheen ${ }^{11}$ & 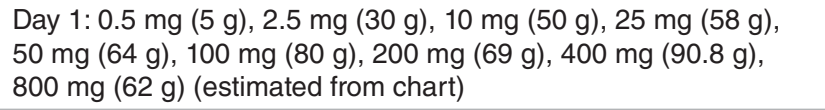 & $\begin{array}{l}\text { Dose response up to about } 100-\mathrm{mg} \\
\text { dose }\end{array}$ \\
\hline Product monograph & 10 mg (64 g), 25 mg (78 g) & Dose response \\
\hline Heise et al. ${ }^{12}$ & Day 1: 10 mg (74 g), 25 mg (90 g), 100 mg (81 g) & Dose response up to $25-\mathrm{mg}$ dose \\
\hline \multicolumn{3}{|l|}{ Dapagliflozin } \\
\hline Parkinson et al. ${ }^{13}$ & $2.5 \mathrm{mg}(37.9 \mathrm{~g}), 5 \mathrm{mg}(45.2 \mathrm{~g}), 10 \mathrm{mg}(86.4 \mathrm{~g})$ & Dose response \\
\hline Yang et al. ${ }^{14}$ & Day 10: 5 mg (28 g), 10 mg (41 g) & Dose response \\
\hline Product monograph & $\begin{array}{l}10 \mathrm{mg}(\sim 70 \mathrm{~g}) \text {; urinary glucose excretion approached a maximum } \\
\text { at } 20-\mathrm{mg} \text { dose }\end{array}$ & Dose response up to 20 -mg dose \\
\hline \multicolumn{3}{|l|}{ Canagliflozin } \\
\hline lijima et al. ${ }^{15}$ & $\begin{array}{l}\text { Ranged from } 80 \text { to } 110 \mathrm{~g} \text {; smallest at } 25-\mathrm{mg} \text { dose, no great } \\
\text { difference at } 100 \text { - to } 400-\mathrm{mg} \text { dose }\end{array}$ & Dose response up to $100-\mathrm{mg}$ dose \\
\hline Devineni and Polidori ${ }^{16}$ & $100-300 \mathrm{mg}$ (ranged from 80 to $120 \mathrm{~g}$ ) & Dose response \\
\hline Product monograph & $100-300 \mathrm{mg}$ (ranged from 77 to $119 \mathrm{~g}$ ) & Not clear \\
\hline Devineni et al. ${ }^{17}$ & 50 mg, 100 mg, 300 mg (increased in a dose-dependent manner) & Dose response \\
\hline \multicolumn{3}{|l|}{ Remogliflozin } \\
\hline Kapur et al. ${ }^{18}$ & $\begin{array}{l}20 \mathrm{mg}(67 \mathrm{mmol}), 50 \mathrm{mg}(97 \mathrm{mmol}), 150 \mathrm{mg}(168 \mathrm{mmol}), 500 \mathrm{mg} \\
(223 \mathrm{mmol}), 1000 \mathrm{mg}(304 \mathrm{mmol})\end{array}$ & Dose response \\
\hline Dobbins et al. ${ }^{19}$ & 200 mg (509 mmol), 1000 mg (918 mmol), 2000 mg (574 mmol) & Dose response up to $1000-\mathrm{mg}$ dose \\
\hline \multicolumn{3}{|l|}{ Ipragliflozin } \\
\hline Veltcamp et al..$^{20}$ & $\begin{array}{l}\text { Dose response was noted up to the } 50-\text { or } 100-\mathrm{mg} \text { dose; actual } \\
\text { change in urinary glucose excretion depended on the study ( } 50 \mathrm{~g} \\
\text { in one study and } 80-90 \mathrm{~g} \text { in another study) }\end{array}$ & Dose response up to $50-\mathrm{mg}$ dose \\
\hline Kadokura et al. ${ }^{21}$ & $50 \mathrm{mg}(80.6 \mathrm{~g} \pm 22.2 \mathrm{~g}), 100 \mathrm{mg}(89.7 \pm 12.3 \mathrm{~g})$ & $\begin{array}{l}\text { No difference between } 50-\mathrm{mg} \text { and } \\
100 \text {-mg dose }\end{array}$ \\
\hline \multicolumn{3}{|l|}{ Ertugliflozin } \\
\hline Amin et al. ${ }^{22}$ & $1 \mathrm{mg}(46.33 \mathrm{~g}), 5 \mathrm{mg}(64.54 \mathrm{~g}), 25 \mathrm{mg}(74.49 \mathrm{~g})$ & Dose response \\
\hline \multicolumn{3}{|l|}{ Tofogliflozin } \\
\hline Ikeda et al. ${ }^{23}$ & $\begin{array}{l}2.5 \mathrm{mg}(217.9 \mathrm{mmol}), 5 \mathrm{mg}(272.3 \mathrm{mmol}), 10 \mathrm{mg}(346.2 \mathrm{mmol}) \text {, } \\
20 \mathrm{mg}(396.0 \mathrm{mmol}), 40 \mathrm{mg}(402.9 \mathrm{mmol})\end{array}$ & Dose response \\
\hline \multicolumn{3}{|l|}{ Sotogliflozin } \\
\hline Zambrowicz et al. ${ }^{24}$ & $400 \mathrm{mg}(29.7 \mathrm{~g})$ & Not clear \\
\hline Rosenstock et al. ${ }^{25}$ & $\begin{array}{l}75 \mathrm{mg}(\sim 18 \mathrm{~g}), 200 \mathrm{mg}(\sim 66 \mathrm{~g}), 400 \mathrm{mg}(55-60 \mathrm{~g}) \text { (estimated } \\
\text { from chart) }\end{array}$ & Dose response up to $200-\mathrm{mg}$ dose \\
\hline
\end{tabular}


dose-response relation between SGLT2 inhibitors and UTI in individuals with type 2 diabetes, relative to other diabetes therapies or placebo.

\section{Methods}

\section{Study design}

This study was designed in accordance with the PRISMA statement on systematic reviews and network meta-analyses ${ }^{26}$ and was registered with PROSPERO (www.crd.york.ac.uk/ prospero/; no. CRD42016038715).

\section{Eligibility criteria}

For this review, we sought to identify RCTs that compared an SGLT2 inhibitor with placebo, with no treatment or with an active antidiabetic control. The SGLT2 inhibitor could be any one of the currently marketed or investigational agents, but we excluded combined SGLT1/SGLT2 inhibitors. An active control could be any of the available oral antidiabetic agents, with the exception of firstgeneration sulphonylureas, because they are rarely used in practice. Patients had to be adults ( $\geq 18 \mathrm{yr}$ ) with type 2 diabetes. The studies had to report on the outcome of UTI, but were not limited by duration of follow-up, year of publication or publication status. Inclusion was limited to studies published in English.

\section{Search strategy}

A health science librarian (M.S.) conducted a comprehensive literature search. The search strategy was developed in the PubMed database (from inception to May 2018) and was then translated for the Cochrane Library via Wiley (from inception to May 2018), Embase via Embase.com (from inception to May 2018) and International Pharmaceutical Abstracts databases via Ebsco (from inception to May 2018). Medical Subject Headings and keyword terms used to capture type 2 diabetes (e.g., "Diabetes Mellitus, Type 2"[Mesh] OR NIDDM[tw] OR t2dm[tw]) were combined with terms relating to SGLT2 inhibitors, including generic names, brand names, chemical names and compound codes as applicable. RCTs were identified with a methodologic search filter. ${ }^{27}$ The librarian also conducted multiple test searches to optimize the sensitivity and specificity of the search parameters. Reference lists of key articles were also screened (by J.R.D.). We identified unpublished (grey literature) RCT data by searching the ProQuest Dissertations \& Theses Global and ClinicalTrials.gov databases. For the various search strategies, see Section 1 of Appendix 1 (available at www.cmajopen.ca/content/6/4/E594/ suppl/DC1).

\section{Study selection and data extraction}

DistillerSR software was used to facilitate a 2-level screening process, first with titles and abstracts and then full text (performed by J.R.D., C.A.G., J.H. and D.C.). We used the "liberal accelerated" method of duplicate screening, ${ }^{28}$ whereby a second reviewer screens only citations that have been rejected by the first reviewer.
For articles included in the review, one reviewer completed the data extraction, and another performed verification (performed by J.R.D., C.A.G. andJ.H.) (for data extraction variables, see Section 2 of Appendix 1). Where gaps existed, the extracted data were supplemented with data from ClinicalTrials.gov. Where data from multiple sources conflicted, information from the published paper was used. Where multiple publications for the same study population existed (e.g., interim analyses or extension studies), the most recent publication was used, except where the most recent publication involved a change in the drug dose.

\section{Assessment of risk of bias}

We used the Cochrane Collaboration domain-based risk assessment tool to identify sources of bias in each study. ${ }^{27}$ This assessment was completed independently by one reviewer, with verification by a second reviewer (performed by J.R.D., C.A.G., J.H.). Each domain was identified as having "low," "high" or "unclear" risk of bias. In addition, the following rules were applied to assign an overall risk of bias: where all domains were considered to have low risk, the overall risk was low; where at least 1 domain was considered to have high risk, the overall risk was high; and where at least 1 domain was considered to have unclear risk (and no domain was considered to have high risk), the overall risk was considered to be unclear. We assessed publication bias using a funnel plot of placebo-controlled trials. ${ }^{27}$

\section{Data synthesis}

We conducted a Bayesian network meta-analysis of RCTs. The doses of SGLT2 inhibitors were categorized into 2 groups: "high dose" and "low dose." These categories were defined on the basis of available marketed doses and urinary glucose excretion rates. Where 2 marketed doses were available for a given drug, the lower dose was categorized as "low" and the higher dose as "high." All other studied doses to the extremes of the 2 marketed doses were categorized in the most proximal dose category. For example, a dose lower than the marketed dose would be considered "low dose." Where 3 marketed doses were available, the middle dose was categorized with the group having the closest urinary glucose excretion rate. For experimental products, categories were defined by looking at the most commonly studied doses and setting a threshold, as was done for the marketed products. We took this approach to avoid placing too much emphasis on ineffective or unsafe doses used in dosefinding studies (for threshold doses, see Section 3 of Appendix 1).

We used a random-effects generalized linear model for binary data, with non-informative priors, to estimate the relative effects, credible intervals and rank probabilities of each of the comparators. We tested convergence of the Markov Chain Monte Carlo simulation (100 000 iterations) with the Gelman-Rubin diagnostic test and used the deviance information criterion to assess model fit. We examined rank probabilities by calculating the surface under the cumulative rank curve values. We tested heterogeneity with the $I^{2}$ statistic for pairwise comparisons and assessed inconsistency by visually comparing the direct and indirect pooled estimates. 
We conducted 3 sensitivity analyses. We altered the threshold between low and high doses to reflect uncertainty in the dose-response relation with urinary glucose excretion. We also restricted the analysis to studies of at least 24 weeks' duration. Finally, we restricted the analysis to studies with a low overall risk of bias.

All of the outcome data were analyzed using the gemtc package of $\mathrm{R}$ statistical software (version 3.4.1).

\section{Ethics approval}

This study was a retrospective analysis of previously published data, and ethics approval was not required.

\section{Results}

In total, 2418 titles and abstracts were screened, and 140 citations met our inclusion criteria. Of these, 35 were excluded because they represented duplicate data (extension studies, post hoc analyses) or because mixed doses or unstable doses were used. A final list of 105 publications was included in the analysis, representing 108 randomized populations (Figure 1), 60082 individuals and 4348 UTIs. Three of the publications ${ }^{29-31}$ reported on more than 1 randomized population (for the reference list of included studies, see Section 4 of Appendix 1).

Most studies examined either dapagliflozin (33 studies), empagliflozin (25 studies), canagliflozin (19 studies) or ipragliflozin (11 studies); 20 studies investigated 1 of 4 other agents (luseogliflozin, remogliflozin, tofogliflozin and ertugliflozin). With respect to comparisons, 4 studies conducted only withinclass comparisons, 89 compared the study drug with placebo, 26 used an active comparator, and 9 included more than 1 unique comparator. Studies ranged from 1 to 208 weeks in duration (for complete study characteristics, see Section 5 of Appendix 1).

We included all of the studies in the first run of our analysis. However, despite 200000 iterations of the Markov Chain Monte Carlo simulation, assessment of the Gelman-Rubin

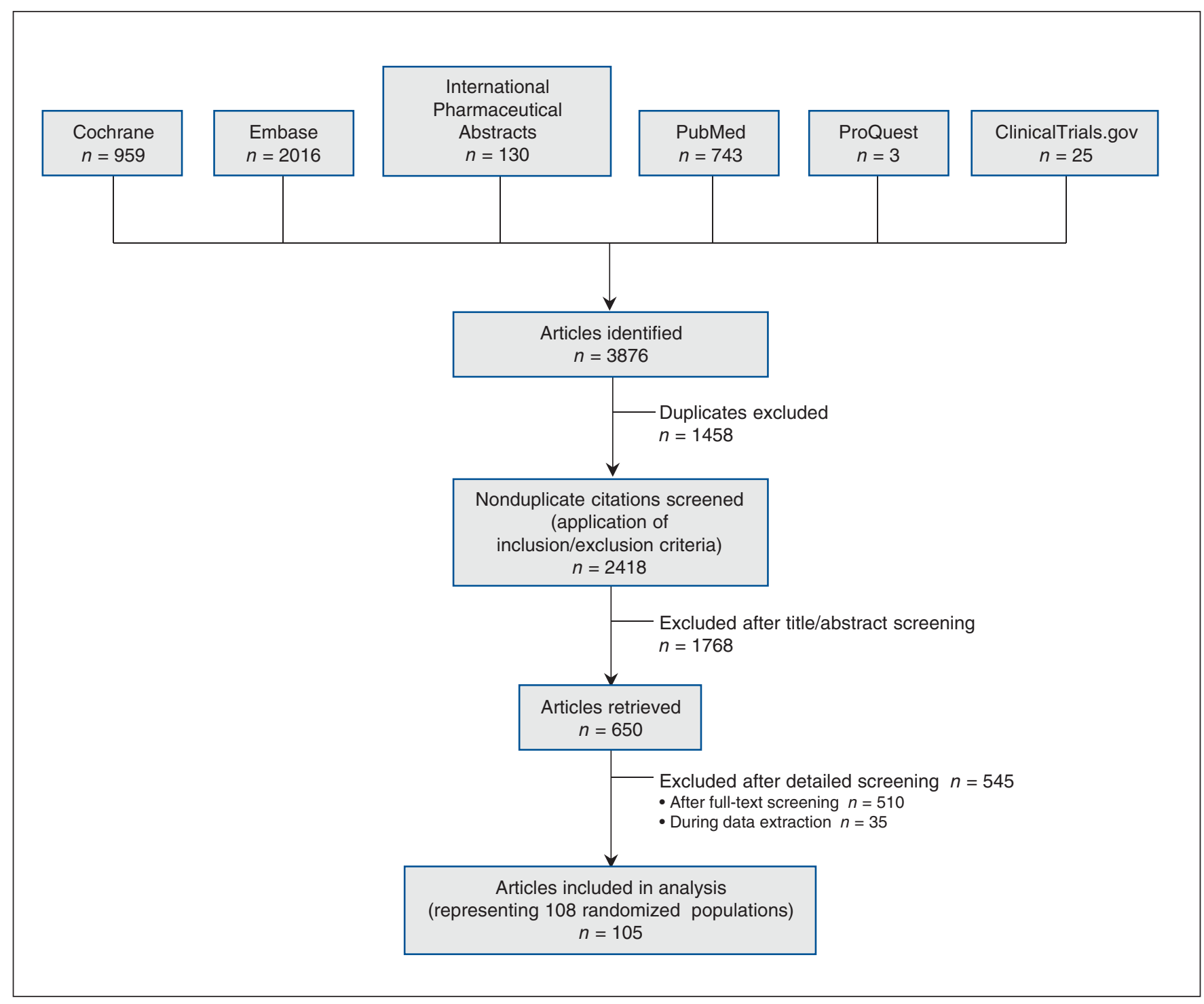

Figure 1: Flow diagram for included studies. Initial database searches were from inception of each particular database to May 2018. 
statistic showed that many nodes did not approach convergence. There were also unexpected protective effects in comparisons that included luseogliflozin. On examination of study results, we found that only 2 cases of UTI were reported across the 4 luseogliflozin studies, each of which was of short duration ( $7 \mathrm{~d}-24 \mathrm{wk})$. After removal of these studies, all nodes approached convergence. The deviance information criterion was also lower, indicating a better model fit. Figure 2 shows the network of available direct evidence without luseogliflozin.

Most comparisons showed a nonsignificant difference in the risk of UTI (Table 2). Exceptions included comparisons of high-dose dapagliflozin $(\geq 10 \mathrm{mg}$ ) with placebo (odds ratio [OR] $1.30,95 \%$ credible interval 1.09-1.57), with active comparators (OR 1.44, 95\% credible interval 1.15-1.79), with empagliflozin at both high doses (OR 1.39, 95\% credible interval 1.12-1.72) and low doses (OR 1.30, 95\% credible interval 1.04-1.60) and with ertugliflozin at low doses (OR $1.43,95 \%$ credible interval 1.01-2.01). Low-dose canagliflozin compared with active comparators also had significantly greater risk (OR 1.29, 95\% credible interval 1.03-1.64). Examination of rank probabilities using surface under the cumulative rank curve values showed results that were consistent with the primary analysis. Specifically, high-dose dapagliflozin was

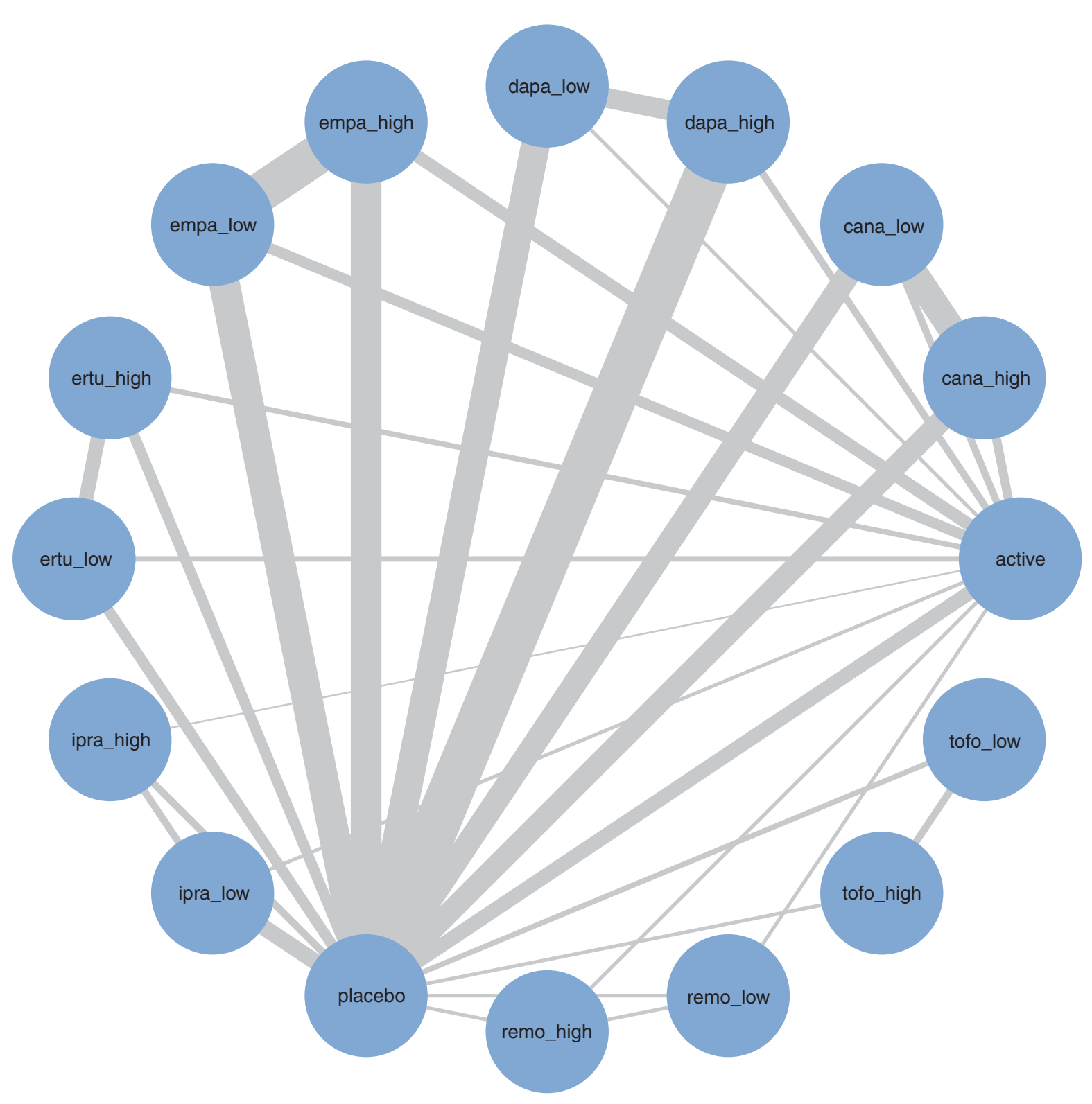

Figure 2: Network diagram for risk of urinary tract infection with sodium glucose cotransporter-2 inhibitors. Note: cana = canagliflozin, dapa = dapagliflozin, empa $=$ empagliflozin, ertu $=$ ertugliflozin, ipra $=$ ipragliflozin, remo $=$ remogliflozin, tofo $=$ tofogliflozin. For each drug, low $=$ low dose, and high = high dose. 
Table 2: Risk of urinary tract infection in association with SGLT2 inhibitor therapy, as reported for network meta-analysis comparisons*

Drug and dose; odds ratio (95\% credible interval)

\begin{tabular}{|c|c|c|c|c|c|c|c|c|c|c|c|c|c|c|c|}
\hline Active & $\begin{array}{l}\text { cana }_{-} \\
\text {high }\end{array}$ & $\underset{\text { cana }}{\text { low }}$ & $\begin{array}{l}\text { dapa } \\
\text { high }\end{array}$ & $\begin{array}{c}\text { dapa } \\
\text { low }\end{array}$ & $\underset{\text { high }}{\text { empa }}$ & $\underset{\text { low }}{\text { empa }}$ & $\begin{array}{l}\text { ertu_ } \\
\text { high }\end{array}$ & $\begin{array}{l}\text { ertu_ } \\
\text { low }\end{array}$ & $\begin{array}{l}\text { ipra } \\
\text { high }\end{array}$ & $\begin{array}{l}\text { ipra } \\
\text { low }\end{array}$ & placebo & $\begin{array}{c}\text { remo__ } \\
\text { high }\end{array}$ & $\begin{array}{c}\text { remo } \\
\text { low }\end{array}$ & $\begin{array}{l}\text { tofo } \\
\text { high }\end{array}$ & $\begin{array}{l}\text { tofo } \\
\text { low }\end{array}$ \\
\hline Active & $\begin{array}{c}1.18 \\
(0.94- \\
1.48)\end{array}$ & $\begin{array}{c}1.29 \\
(1.03- \\
1.64)\end{array}$ & $\begin{array}{c}1.44 \\
(1.15- \\
1.79)\end{array}$ & $\begin{array}{c}1.20 \\
(0.92- \\
1.55)\end{array}$ & $\begin{array}{c}1.03 \\
(0.87- \\
1.24)\end{array}$ & $\begin{array}{c}1.11 \\
(0.92- \\
1.34)\end{array}$ & $\begin{array}{c}1.20 \\
(0.90- \\
1.61)\end{array}$ & $\begin{array}{c}1.00 \\
(0.75- \\
1.35)\end{array}$ & $\begin{array}{c}1.11 \\
(0.56- \\
2.21)\end{array}$ & $\begin{array}{c}1.11 \\
(0.63- \\
2.07)\end{array}$ & $\begin{array}{c}1.10 \\
(0.93- \\
1.31)\end{array}$ & $\begin{array}{c}0.81 \\
(0.26- \\
2.60)\end{array}$ & $\begin{array}{c}1.11 \\
(0.36- \\
3.62)\end{array}$ & $\begin{array}{c}1.68 \\
(0.26- \\
13.09)\end{array}$ & $\begin{array}{c}1.62 \\
(0.30- \\
10.50)\end{array}$ \\
\hline $\begin{array}{l}0.84 \\
(0.67- \\
1.06)\end{array}$ & $\begin{array}{l}\text { cana } \\
\text { high }\end{array}$ & $\begin{array}{c}1.10 \\
(0.90- \\
1.33)\end{array}$ & $\begin{array}{c}1.21 \\
(0.92- \\
1.56)\end{array}$ & $\begin{array}{c}1.01 \\
(0.74- \\
1.36)\end{array}$ & $\begin{array}{c}0.87 \\
(0.68- \\
1.10)\end{array}$ & $\begin{array}{c}0.94 \\
(0.73- \\
1.18)\end{array}$ & $\begin{array}{c}1.01 \\
(0.73- \\
1.45)\end{array}$ & $\begin{array}{c}0.85 \\
(0.60- \\
1.21)\end{array}$ & $\begin{array}{c}0.93 \\
(0.46- \\
1.95)\end{array}$ & $\begin{array}{c}0.94 \\
(0.52- \\
1.78)\end{array}$ & $\begin{array}{c}0.93 \\
(0.75- \\
1.15)\end{array}$ & $\begin{array}{c}0.68 \\
(0.21- \\
2.27)\end{array}$ & $\begin{array}{c}0.93 \\
(0.30- \\
3.06)\end{array}$ & $\begin{array}{c}1.41 \\
(0.22- \\
11.37)\end{array}$ & $\begin{array}{c}1.36 \\
(0.26- \\
9.43)\end{array}$ \\
\hline $\begin{array}{l}0.77 \\
(0.61- \\
0.97)\end{array}$ & $\begin{array}{c}0.91 \\
(0.75- \\
1.11)\end{array}$ & $\begin{array}{c}\text { cana } \\
\text { low }\end{array}$ & $\begin{array}{c}1.11 \\
(0.84- \\
1.45)\end{array}$ & $\begin{array}{c}0.92 \\
(0.68- \\
1.25)\end{array}$ & $\begin{array}{c}0.80 \\
(0.63- \\
1.01)\end{array}$ & $\begin{array}{c}0.86 \\
(0.67- \\
1.09)\end{array}$ & $\begin{array}{c}0.92 \\
(0.66- \\
1.32)\end{array}$ & $\begin{array}{c}0.77 \\
(0.54- \\
1.10)\end{array}$ & $\begin{array}{c}0.86 \\
(0.41- \\
1.79)\end{array}$ & $\begin{array}{c}0.86 \\
(0.47- \\
1.64)\end{array}$ & $\begin{array}{c}0.85 \\
(0.68- \\
1.06)\end{array}$ & $\begin{array}{c}0.62 \\
(0.19- \\
2.06)\end{array}$ & $\begin{array}{c}0.85 \\
(0.27- \\
2.83)\end{array}$ & $\begin{array}{c}1.28 \\
(0.20- \\
10.40)\end{array}$ & $\begin{array}{c}1.24 \\
(0.24- \\
8.50)\end{array}$ \\
\hline $\begin{array}{l}0.70 \\
(0.56- \\
0.87)\end{array}$ & $\begin{array}{c}0.82 \\
(0.64- \\
1.09)\end{array}$ & $\begin{array}{c}0.90 \\
(0.69- \\
1.20)\end{array}$ & $\begin{array}{c}\text { dapa }_{-} \\
\text {high }\end{array}$ & $\begin{array}{c}0.83 \\
(0.66- \\
1.05)\end{array}$ & $\begin{array}{c}0.72 \\
(0.58- \\
0.89)\end{array}$ & $\begin{array}{c}0.77 \\
(0.62- \\
0.96)\end{array}$ & $\begin{array}{c}0.84 \\
(0.60- \\
1.19)\end{array}$ & $\begin{array}{c}0.70 \\
(0.50- \\
0.99)\end{array}$ & $\begin{array}{c}0.77 \\
(0.38- \\
1.57)\end{array}$ & $\begin{array}{c}0.78 \\
(0.43- \\
1.44)\end{array}$ & $\begin{array}{c}0.77 \\
(0.64- \\
0.92)\end{array}$ & $\begin{array}{c}0.56 \\
(0.18- \\
1.86)\end{array}$ & $\begin{array}{c}0.77 \\
(0.25- \\
2.57)\end{array}$ & $\begin{array}{c}1.16 \\
(0.18- \\
9.72)\end{array}$ & $\begin{array}{c}1.13 \\
(0.21- \\
7.88)\end{array}$ \\
\hline $\begin{array}{l}0.84 \\
(0.65- \\
1.09)\end{array}$ & $\begin{array}{c}0.99 \\
(0.74- \\
1.36)\end{array}$ & $\begin{array}{c}1.08 \\
(0.80- \\
1.48)\end{array}$ & $\begin{array}{c}1.20 \\
(0.95- \\
1.50)\end{array}$ & $\begin{array}{c}\text { dapa } \\
\text { low }\end{array}$ & $\begin{array}{c}0.86 \\
(0.67- \\
1.13)\end{array}$ & $\begin{array}{c}0.92 \\
(0.72- \\
1.22)\end{array}$ & $\begin{array}{c}1.01 \\
(0.70- \\
1.47)\end{array}$ & $\begin{array}{c}0.84 \\
(0.59- \\
1.21)\end{array}$ & $\begin{array}{c}0.93 \\
(0.44- \\
1.94)\end{array}$ & $\begin{array}{c}0.93 \\
(0.50- \\
1.77)\end{array}$ & $\begin{array}{c}0.92 \\
(0.73- \\
1.17)\end{array}$ & $\begin{array}{c}0.68 \\
(0.21- \\
2.18)\end{array}$ & $\begin{array}{c}0.92 \\
(0.29- \\
3.12)\end{array}$ & $\begin{array}{c}1.40 \\
(0.22- \\
11.90)\end{array}$ & $\begin{array}{c}1.34 \\
(0.25- \\
10.04)\end{array}$ \\
\hline $\begin{array}{l}0.97 \\
(0.81- \\
1.15)\end{array}$ & $\begin{array}{c}1.14 \\
(0.91- \\
1.46)\end{array}$ & $\begin{array}{c}1.25 \\
(0.99- \\
1.59)\end{array}$ & $\begin{array}{c}1.39 \\
(1.12- \\
1.72)\end{array}$ & $\begin{array}{c}1.16 \\
(0.88- \\
1.50)\end{array}$ & $\begin{array}{c}\text { empa } \\
\text { high }\end{array}$ & $\begin{array}{c}1.07 \\
(0.95- \\
1.23)\end{array}$ & $\begin{array}{c}1.16 \\
(0.87- \\
1.59)\end{array}$ & $\begin{array}{c}0.97 \\
(0.71- \\
1.32)\end{array}$ & $\begin{array}{c}1.07 \\
(0.53- \\
2.17)\end{array}$ & $\begin{array}{c}1.08 \\
(0.61- \\
1.99)\end{array}$ & $\begin{array}{c}1.06 \\
(0.94- \\
1.22)\end{array}$ & $\begin{array}{c}0.78 \\
(0.25- \\
2.58)\end{array}$ & $\begin{array}{c}1.07 \\
(0.34- \\
3.53)\end{array}$ & $\begin{array}{c}1.62 \\
(0.26- \\
13.17)\end{array}$ & $\begin{array}{c}1.56 \\
(0.29- \\
10.59)\end{array}$ \\
\hline $\begin{array}{l}0.90 \\
(0.74- \\
1.08)\end{array}$ & $\begin{array}{c}1.07 \\
(0.84- \\
1.36)\end{array}$ & $\begin{array}{c}1.17 \\
(0.92- \\
1.49)\end{array}$ & $\begin{array}{c}1.30 \\
(1.04- \\
1.60)\end{array}$ & $\begin{array}{c}1.08 \\
(0.82- \\
1.40)\end{array}$ & $\begin{array}{c}0.93 \\
(0.82- \\
1.06)\end{array}$ & $\underset{\text { low }}{\text { empa }_{-}}$ & $\begin{array}{c}1.08 \\
(0.80- \\
1.48)\end{array}$ & $\begin{array}{c}0.91 \\
(0.66- \\
1.23)\end{array}$ & $\begin{array}{c}0.99 \\
(0.49- \\
2.04)\end{array}$ & $\begin{array}{c}1.00 \\
(0.57- \\
1.86)\end{array}$ & $\begin{array}{c}0.99 \\
(0.87- \\
1.13)\end{array}$ & $\begin{array}{c}0.73 \\
(0.23- \\
2.44)\end{array}$ & $\begin{array}{c}1.00 \\
(0.32- \\
3.30)\end{array}$ & $\begin{array}{c}1.50 \\
(0.24- \\
12.11)\end{array}$ & $\begin{array}{c}1.45 \\
(0.27- \\
9.83)\end{array}$ \\
\hline $\begin{array}{l}0.83 \\
(0.62- \\
1.11)\end{array}$ & $\begin{array}{c}0.99 \\
(0.69- \\
1.37)\end{array}$ & $\begin{array}{c}1.08 \\
(0.76- \\
1.52)\end{array}$ & $\begin{array}{c}1.19 \\
(0.84- \\
1.67)\end{array}$ & $\begin{array}{c}0.99 \\
(0.68- \\
1.43)\end{array}$ & $\begin{array}{c}0.86 \\
(0.63- \\
1.15)\end{array}$ & $\begin{array}{c}0.93 \\
(0.68- \\
1.24)\end{array}$ & $\begin{array}{l}\text { ertu_ } \\
\text { high }\end{array}$ & $\begin{array}{c}0.83 \\
(0.63- \\
1.11)\end{array}$ & $\begin{array}{c}0.92 \\
(0.44- \\
1.95)\end{array}$ & $\begin{array}{c}0.93 \\
(0.51- \\
1.82)\end{array}$ & $\begin{array}{c}0.92 \\
(0.68- \\
1.22)\end{array}$ & $\begin{array}{c}0.67 \\
(0.21- \\
2.28)\end{array}$ & $\begin{array}{c}0.92 \\
(0.286- \\
3.112)\end{array}$ & $\begin{array}{c}1.40 \\
(0.22- \\
11.11)\end{array}$ & $\begin{array}{c}1.35 \\
(0.24- \\
9.24)\end{array}$ \\
\hline $\begin{array}{l}0.99 \\
(0.74- \\
1.34)\end{array}$ & $\begin{array}{c}1.18 \\
(0.83- \\
1.67)\end{array}$ & $\begin{array}{c}1.29 \\
(0.91- \\
1.85)\end{array}$ & $\begin{array}{c}1.43 \\
(1.01- \\
2.01)\end{array}$ & $\begin{array}{c}1.20 \\
(0.82- \\
1.70)\end{array}$ & $\begin{array}{c}1.03 \\
(0.76- \\
1.41)\end{array}$ & $\begin{array}{c}1.10 \\
(0.82- \\
1.52)\end{array}$ & $\begin{array}{c}1.20 \\
(0.90- \\
1.58)\end{array}$ & $\begin{array}{l}\text { ertu_ } \\
\text { low }\end{array}$ & $\begin{array}{c}1.12 \\
(0.52- \\
2.29)\end{array}$ & $\begin{array}{c}1.11 \\
(0.61- \\
2.20)\end{array}$ & $\begin{array}{c}1.10 \\
(0.82- \\
1.47)\end{array}$ & $\begin{array}{c}0.81 \\
(0.25- \\
2.66)\end{array}$ & $\begin{array}{c}1.09 \\
(0.35- \\
3.65)\end{array}$ & $\begin{array}{c}1.67 \\
(0.26- \\
13.35)\end{array}$ & $\begin{array}{c}1.60 \\
(0.29- \\
11.40)\end{array}$ \\
\hline $\begin{array}{l}0.90 \\
(0.45- \\
1.80)\end{array}$ & $\begin{array}{c}1.08 \\
(0.51- \\
2.20)\end{array}$ & $\begin{array}{c}1.17 \\
(0.56- \\
2.42)\end{array}$ & $\begin{array}{c}1.29 \\
(0.64- \\
2.65)\end{array}$ & $\begin{array}{c}1.07 \\
(0.52- \\
2.26)\end{array}$ & $\begin{array}{c}0.94 \\
(0.46- \\
1.88)\end{array}$ & $\begin{array}{c}1.01 \\
(0.49- \\
2.04)\end{array}$ & $\begin{array}{c}1.09 \\
(0.51- \\
2.26)\end{array}$ & $\begin{array}{c}0.90 \\
(0.44- \\
1.90)\end{array}$ & $\begin{array}{l}\text { ipra } \\
\text { high }\end{array}$ & $\begin{array}{c}1.01 \\
(0.54- \\
1.92)\end{array}$ & $\begin{array}{c}1.00 \\
(0.50- \\
2.00)\end{array}$ & $\begin{array}{c}0.72 \\
(0.20- \\
3.13)\end{array}$ & $\begin{array}{c}0.97 \\
(0.27- \\
4.11)\end{array}$ & $\begin{array}{c}1.56 \\
(0.21- \\
12.19)\end{array}$ & $\begin{array}{c}1.52 \\
(0.23- \\
10.89)\end{array}$ \\
\hline $\begin{array}{l}0.90 \\
(0.48- \\
1.60)\end{array}$ & $\begin{array}{c}1.06 \\
(0.56- \\
1.92)\end{array}$ & $\begin{array}{c}1.16 \\
(0.61- \\
2.12)\end{array}$ & $\begin{array}{c}1.29 \\
(0.69- \\
2.31)\end{array}$ & $\begin{array}{c}1.07 \\
(0.57- \\
1.99)\end{array}$ & $\begin{array}{c}0.93 \\
(0.50- \\
1.65)\end{array}$ & $\begin{array}{c}1.00 \\
(0.54- \\
1.76)\end{array}$ & $\begin{array}{c}1.07 \\
(0.55- \\
1.97)\end{array}$ & $\begin{array}{c}0.90 \\
(0.46- \\
1.65)\end{array}$ & $\begin{array}{c}0.99 \\
(0.52- \\
1.87)\end{array}$ & $\begin{array}{l}\text { ipra } \\
\text { low }\end{array}$ & $\begin{array}{c}0.99 \\
(0.54- \\
1.75)\end{array}$ & $\begin{array}{c}0.72 \\
(0.21- \\
2.73)\end{array}$ & $\begin{array}{c}0.98 \\
(0.29- \\
3.73)\end{array}$ & $\begin{array}{c}1.53 \\
(0.22- \\
11.92)\end{array}$ & $\begin{array}{c}1.48 \\
(0.25- \\
9.62)\end{array}$ \\
\hline $\begin{array}{l}0.91 \\
(0.76- \\
1.07)\end{array}$ & $\begin{array}{c}1.08 \\
(0.87- \\
1.34)\end{array}$ & $\begin{array}{c}1.17 \\
(0.94- \\
1.47)\end{array}$ & $\begin{array}{c}1.30 \\
(1.09- \\
1.57)\end{array}$ & $\begin{array}{c}1.08 \\
(0.86- \\
1.38)\end{array}$ & $\begin{array}{c}0.94 \\
(0.82- \\
1.06)\end{array}$ & $\begin{array}{c}1.01 \\
(0.88- \\
1.15)\end{array}$ & $\begin{array}{c}1.09 \\
(0.82- \\
1.47)\end{array}$ & $\begin{array}{c}0.91 \\
(0.68- \\
1.22)\end{array}$ & $\begin{array}{c}1.00 \\
(0.50- \\
2.01)\end{array}$ & $\begin{array}{c}1.01 \\
(0.57- \\
1.85)\end{array}$ & placebo & $\begin{array}{c}0.73 \\
(0.23- \\
2.42)\end{array}$ & $\begin{array}{c}1.00 \\
(0.32- \\
3.30)\end{array}$ & $\begin{array}{c}1.51 \\
(0.24- \\
12.14)\end{array}$ & $\begin{array}{c}1.47 \\
(0.28- \\
9.95)\end{array}$ \\
\hline $\begin{array}{l}1.23 \\
(0.38- \\
3.93)\end{array}$ & $\begin{array}{c}1.46 \\
(0.44- \\
4.75)\end{array}$ & $\begin{array}{c}1.61 \\
(0.48- \\
5.14)\end{array}$ & $\begin{array}{c}1.77 \\
(0.54- \\
5.56)\end{array}$ & $\begin{array}{c}1.48 \\
(0.46- \\
4.80)\end{array}$ & $\begin{array}{c}1.28 \\
(0.39- \\
4.07)\end{array}$ & $\begin{array}{c}1.37 \\
(0.41- \\
4.34)\end{array}$ & $\begin{array}{c}1.49 \\
(0.44- \\
4.86)\end{array}$ & $\begin{array}{c}1.24 \\
(0.38- \\
4.04)\end{array}$ & $\begin{array}{c}1.39 \\
(0.32- \\
4.93)\end{array}$ & $\begin{array}{c}1.38 \\
(0.37- \\
4.80)\end{array}$ & $\begin{array}{c}1.36 \\
(0.41- \\
4.28)\end{array}$ & $\begin{array}{c}\text { remo_- } \\
\text { high }\end{array}$ & $\begin{array}{c}1.34 \\
(0.44- \\
4.11)\end{array}$ & $\begin{array}{c}2.08 \\
(0.24- \\
22.26)\end{array}$ & $\begin{array}{c}1.92 \\
(0.26- \\
20.93)\end{array}$ \\
\hline $\begin{array}{l}0.90 \\
(0.28- \\
2.79)\end{array}$ & $\begin{array}{c}1.08 \\
(0.33- \\
3.40)\end{array}$ & $\begin{array}{c}1.18 \\
(0.35- \\
3.68)\end{array}$ & $\begin{array}{c}1.30 \\
(0.39- \\
3.97)\end{array}$ & $\begin{array}{c}1.09 \\
(0.32- \\
3.50)\end{array}$ & $\begin{array}{c}0.93 \\
(0.28- \\
2.90)\end{array}$ & $\begin{array}{c}1.00 \\
(0.30- \\
3.12)\end{array}$ & $\begin{array}{c}1.09 \\
(0.32- \\
3.50)\end{array}$ & $\begin{array}{c}0.92 \\
(0.27- \\
2.89)\end{array}$ & $\begin{array}{c}1.03 \\
(0.24- \\
3.74)\end{array}$ & $\begin{array}{c}1.02 \\
(0.27- \\
3.41)\end{array}$ & $\begin{array}{c}1.0 \\
(0.30- \\
3.08)\end{array}$ & $\begin{array}{c}0.75 \\
(0.24- \\
2.28)\end{array}$ & $\begin{array}{c}\text { remo } \\
\text { low }\end{array}$ & $\begin{array}{c}1.50 \\
(0.17- \\
16.43)\end{array}$ & $\begin{array}{c}1.42 \\
(0.19- \\
14.58)\end{array}$ \\
\hline $\begin{array}{l}0.60 \\
(0.08- \\
3.85)\end{array}$ & $\begin{array}{c}0.71 \\
(0.09- \\
4.64)\end{array}$ & $\begin{array}{c}0.78 \\
(0.10- \\
4.95)\end{array}$ & $\begin{array}{c}0.86 \\
(0.10- \\
5.51)\end{array}$ & $\begin{array}{c}0.72 \\
(0.08- \\
4.62)\end{array}$ & $\begin{array}{c}0.62 \\
(0.08- \\
3.89)\end{array}$ & $\begin{array}{c}0.66 \\
(0.08- \\
4.18)\end{array}$ & $\begin{array}{c}0.71 \\
(0.09- \\
4.57)\end{array}$ & $\begin{array}{c}0.60 \\
(0.08- \\
3.82)\end{array}$ & $\begin{array}{c}0.64 \\
(0.08- \\
4.83)\end{array}$ & $\begin{array}{c}0.65 \\
(0.08- \\
4.54)\end{array}$ & $\begin{array}{c}0.66 \\
(0.08- \\
4.14)\end{array}$ & $\begin{array}{c}0.48 \\
(0.04- \\
4.21)\end{array}$ & $\begin{array}{c}0.67 \\
(0.06- \\
5.94)\end{array}$ & $\begin{array}{l}\text { tofo- } \\
\text { high }\end{array}$ & $\begin{array}{c}0.98 \\
(0.40- \\
2.37)\end{array}$ \\
\hline $\begin{array}{l}0.62 \\
(0.10- \\
3.31)\end{array}$ & $\begin{array}{c}0.74 \\
(0.11- \\
3.93)\end{array}$ & $\begin{array}{c}0.81 \\
(0.12- \\
4.21)\end{array}$ & $\begin{array}{c}0.89 \\
(0.13- \\
4.76)\end{array}$ & $\begin{array}{c}0.74 \\
(0.10- \\
4.02)\end{array}$ & $\begin{array}{c}0.64 \\
(0.09- \\
3.41)\end{array}$ & $\begin{array}{c}0.69 \\
(0.10- \\
3.66)\end{array}$ & $\begin{array}{c}0.74 \\
(0.11- \\
4.14)\end{array}$ & $\begin{array}{c}0.62 \\
(0.09- \\
3.41)\end{array}$ & $\begin{array}{c}0.66 \\
(0.09- \\
4.39)\end{array}$ & $\begin{array}{c}0.68 \\
(0.10- \\
4.06)\end{array}$ & $\begin{array}{c}0.68 \\
(0.10- \\
3.59)\end{array}$ & $\begin{array}{c}0.52 \\
(0.05- \\
3.91)\end{array}$ & $\begin{array}{c}0.70 \\
(0.07- \\
5.29)\end{array}$ & $\begin{array}{c}1.02 \\
(0.42- \\
2.52)\end{array}$ & $\begin{array}{l}\text { tofo } \\
\text { low }\end{array}$ \\
\hline
\end{tabular}

the least favourable and high-dose remogliflozin and active comparators (grouped) were the most favourable with respect to risk of UTI (for the forest plot of placebo treatment comparisons and the list of surface under the cumulative rank curve values, see Section 6 of Appendix 1).

Examination of the $I^{2}$ value for each of the comparisons showed homogeneity, with most values of $I^{2}$ at $0 \%$ (and all $<45 \%$ ). When we back-calculated indirect risk estimates and compared them with direct evidence to assess for consistency, we found no major discrepancies between the estimates, which suggested that the consistency assumption was met (for the complete list of pairwise indirect and pooled estimates, see Section 7 of Appendix 1).

\section{Risk of bias}

Generally, the studies were of high methodologic quality. The overall quality assessment indicated that more than half of the studies (54 or 51\%) were at low risk of bias. About one-third (32 or $30 \%$ ) had unclear reporting of randomization sequence, and one-quarter ( 26 or $25 \%$ ) had unclear or high risk of bias for blinded outcome assessment (Figure 3). No indication of publication bias was observed in the funnel plot (Section 8 of Appendix 1).

\section{Sensitivity analysis}

The results of the sensitivity analyses were consistent with those of the primary analysis. When the threshold between 


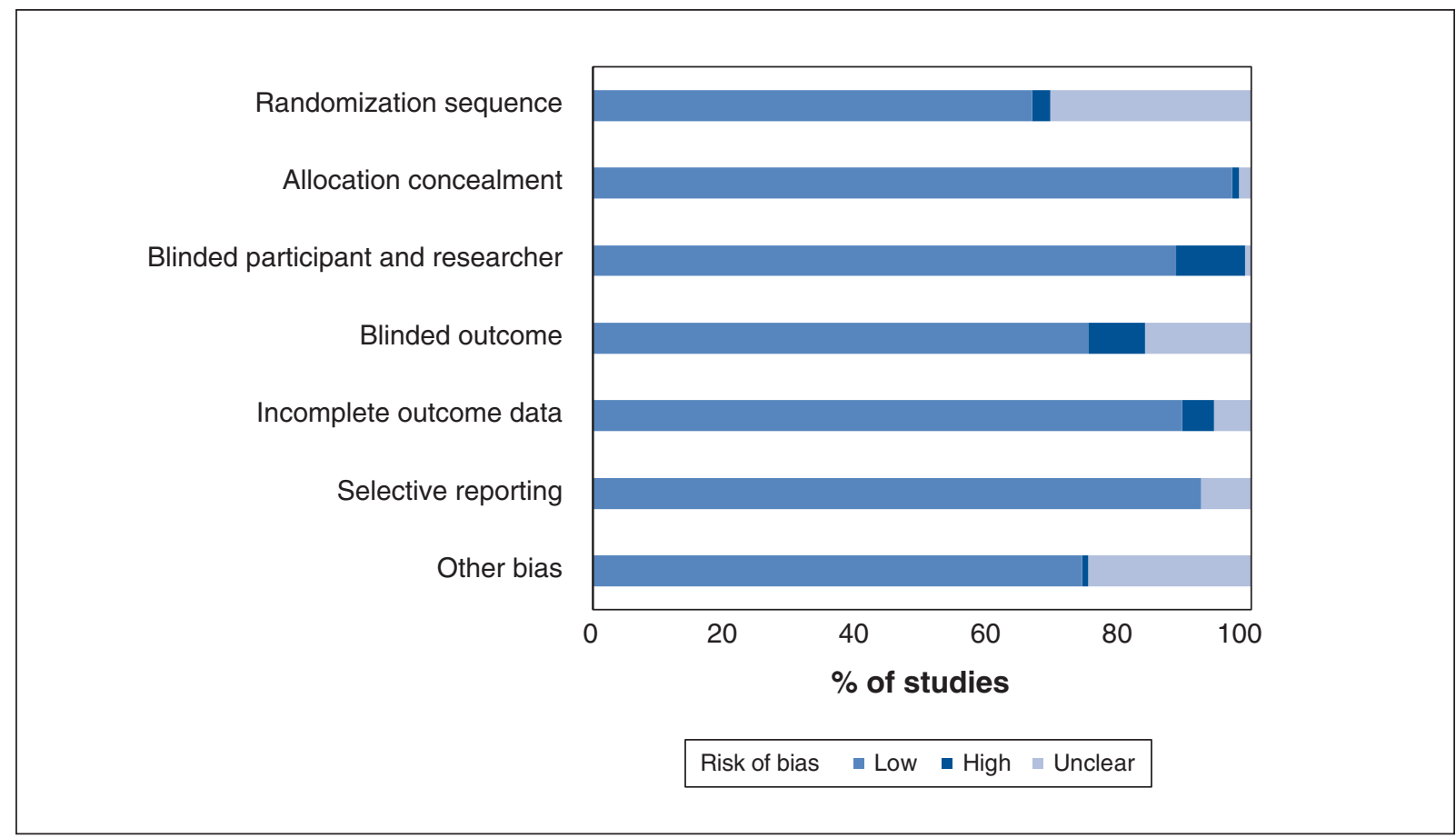

Figure 3: Assessment of risk of bias according to various domains ( $n=105$ studies).

high and low doses was altered, high-dose dapagliflozin still showed an increased risk of UTI compared with placebo, active comparators and high-dose empagliflozin, but also showed an increased risk relative to low doses of ipragliflozin and ertugliflozin. The thresholds for dapagliflozin doses were not adjusted in this sensitivity analysis, because an alternative definition was not suitable. Ipragliflozin at low doses showed a significantly lower risk of UTI than high doses of canagliflozin, ertugliflozin, ipragliflozin and dapagliflozin.

In the analysis of studies lasting 24 weeks or longer, fewer comparisons among experimental agents were possible. However, the findings were consistent with those of the primary analysis, whereby high-dose dapagliflozin had a high risk compared with placebo, active comparator and empagliflozin. Restriction of the analysis to studies with an overall low risk of bias $(n=57)$ resulted in no significant differences among the drug regimens.

In each of the sensitivity analyses, there were treatment arms with insufficient data to accurately estimate risk (for complete results of the sensitivity analyses, see Section 9 of Appendix 1).

\section{Interpretation}

The main findings of this study suggest no dose-response association between SGLT2 inhibitors and UTI risk; however, dapagliflozin (at doses $\geq 10 \mathrm{mg}$ ) appears to be an exception to this general finding. Specifically, high-dose dapagliflozin compared with placebo, active comparators and empagliflozin was associated with a small increase in the risk of UTI.

Several other meta-analyses have reported on the association between SGLT2 inhibitors and UTIs, ${ }^{7,8,32-38}$ with incon- sistent results, including increased risk with dapagliflozin, ${ }^{7,8,33}$ increased risk with SGLT2 inhibitors, ${ }^{34,35}$ and no difference in risk. ${ }^{36-38}$ However, given the continuing postmarketing surveillance of these new agents, new RCTs are being published rapidly, and these previous meta-analyses are quickly becoming outdated. ${ }^{34,37}$ In addition, several studies have applied additional eligibility criteria, such as including only marketed agents, ${ }^{33}$ placebo comparison trials ${ }^{33}$ or studies of a certain duration (e.g., > $24 \mathrm{wk}$ ). ${ }^{7,8,33}$ The largest meta-analysis to date, which pooled results from 86 RCTs representing 50880 patients, found no increased risk of UTIs (relative risk 1.03, $95 \%$ confidence interval $0.96-1.11) .{ }^{38}$ Subgroup analysis by dose in this previous study also showed an increased risk only among users of dapagliflozin at a $10-\mathrm{mg}$ dose.

A mechanism for the increased risk of UTI with dapagliflozin is not clear; however, there is variation in the pharmacokinetic and pharmacodynamic profiles of individual SGLT2 inhibitors. The SGLT2 inhibitors have shown a positive doseeffect relation with urinary glucose excretion, but this appears to have a ceiling effect with several agents. Maximum effects have been documented at about the starting doses for empagliflozin $(10 \mathrm{mg})^{9,12}$ and canagliflozin $(100 \mathrm{mg}),{ }^{15-17}$ but continued through the dosing range with dapagliflozin. ${ }^{13}$ This may explain why the current study showed a dose-dependent relation for UTIs with dapagliflozin. It is unclear why an increased risk of UTI was observed with low-dose canagliflozin. Our sensitivity analysis showed a potential decreased risk of UTI among users of low-dose ipragliflozin ( $\leq 50 \mathrm{mg}$ ) relative to those using high doses of canagliflozin, empagliflozin, ertugliflozin or ipragliflozin; high and low doses of dapagliflozin; or placebo. Pharmacodynamic evidence for ipragliflozin has been variable, with inconsistent estimates of 
the degree of urinary glucose excretion and the dose-response relation. However, there is also no indication that ipragliflozin is unique in any way that would support a physiologic mechanism for the decreased risk of UTI. Further work is needed to examine this finding.

Our findings are consistent with previous findings supporting a lack of compelling data that would suggest a class effect in terms of UTI risk. Our study also extends the evidence by including additional studies, which has resulted in a more precise effect estimate. This study included as many studies as we could find to investigate dose response encompassing both marketed and nonmarketed agents, and active and inactive comparators in studies of any duration.

\section{Limitations}

This systematic review of the association between SGLT2 inhibitors and UTIs had some limitations. The outcome of UTI is very well reported, but we did not identify data on the progression of UTI to more serious infections. This gap in reporting makes it impossible to support or refute the concern that SGLT2 inhibitors may lead to serious infections. It is already known that, as a population, patients with diabetes have an increased risk of infections of all origins. ${ }^{39}$ The 19 serious cases of UTI associated with SGLT2 inhibitors reported in the United States 6 may be a result of increased vigilance for newly marketed drugs. The role of urinary glucose excretion in the pathogenesis of urinary tract infections is not well characterized. It has been postulated that increased urinary glucose excretion may not directly cause infections but rather may create a rich environment for bacterial growth and affect bacterial adherence to uroepithelial cells..$^{4-42}$ Because of the volume of studies included, it was not feasible to contact authors regarding these data. Other limitations included restriction of the analysis to studies published in English, and verification of data abstraction and bias assessment by a second reviewer, rather than independent duplication of abstraction and assessment. Finally, we found no study that compared 2 different SGLT2 inhibitors in a single trial; therefore, the strength of evidence for comparisons between SGLT2 inhibitors is weak.

\section{Conclusion}

Current evidence does not support a dose-response risk profile for UTIs with SGLT2 inhibitors as a class. Although high doses of dapagliflozin $(\geq 10 \mathrm{mg})$ did appear to be associated with increased risk, this risk was attenuated in an analysis restricted to RCTs with low risk of bias. Further studies are needed to quantify the association between SGLT2 inhibitors and more serious infections such as pyelonephritis.

\section{References}

1. Zinman B, Wanner C, Lachin JM, et al.; EMPA-REG OUTCOME Investigators. Empagliflozin, cardiovascular outcomes, and mortality in type 2 diabetes. N Engl 7 Med 2015;373:2117-28.

2. Neal B, Perkovic V, Mahaffey KW, et al.; CANVAS Program Collaborative Group. Canagliflozin and cardiovascular and renal events in type 2 diabetes. N Engl 7 Med 2017;377:644-57.

3. Canadian Diabetes Association Clinical Practice Guidelines Expert Committee; Harper W, Clement M, Goldenberg R, et al. Pharmacologic management of type 2 diabetes. Can 7 Diabetes 2013;37(Suppl 1):S61-8.
4. Komoroski B, Vachharajani N, Boulton D, et al. Dapagliflozin, a novel SGLT2 inhibitor, induces dose-dependent glucosuria in healthy subjects. Clin Pharmacol Ther 2009;85:520-6.

5. Geerlings S, Fonseca V, Castro-Diaz D, et al. Genital and urinary tract infections in diabetes: impact of pharmacologically-induced glucosuria. Diabetes Res Clin Pract 2014;103:373-81.

6. FDA drug safety communication: FDA revises labels of SGLT2 inhibitors for diabetes to include warnings about too much acid in the blood and serious urinary tract infections. Silver Spring (MD): US Food and Drug Administration; 2015. Available: www.fda.gov/Drugs/DrugSafety/ucm475463.htm (accessed 2015 Dec. 10).

7. Liu J, Li L, Li S, et al. Effects of SGLT2 inhibitors on UTIs and genital infections in type 2 diabetes mellitus: a systematic review and meta-analysis. Sci Rep 2017;7:2824.

8. Li D, Wang T, Shen S, et al. Urinary tract and genital infections in patients with type 2 diabetes treated with sodium-glucose co-transporter 2 inhibitors: a meta-analysis of randomized controlled trials. Diabetes Obes Metab 2017;19: 348-55.

9. Zhao X, Cui Y, Zhao S, et al. Pharmacokinetic and pharmacodynamic properties and tolerability of single- and multiple-dose once-daily empagliflozin, a sodium glucose cotransporter 2 inhibitor, in Chinese patients with type 2 diabetes mellitus. Clin Ther 2015;37:1493-502.

10. Kanada S, Koiwai K, Taniguchi A, et al. Pharmacokinetics, pharmacodynamics, safety and tolerability of 4 weeks' treatment with empagliflozin in Japanese patients with type 2 diabetes mellitus. 7 Diabetes Investig 2013;4:613-7.

11. Scheen AJ. Pharmacokinetic and pharmacodynamic profile of empagliflozin, a sodium glucose co-transporter 2 inhibitor. Clin Pharmacokinet 2014;53: 213-25.

12. Heise T, Seewaldt-Becker E, Macha S, et al. Safety, tolerability, pharmacokinetics and pharmacodynamics following 4 weeks' treatment with empagliflozin once daily in patients with type 2 diabetes. Diabetes Obes Metab 2013;15:613-21.

13. Parkinson J, Tang W, Johansson CC, et al. Comparison of the exposureresponse relationship of dapagliflozin in adult and paediatric patients with type 2 diabetes mellitus. Diabetes Obes Metab 2016;18:685-92.

14. Yang W, Han P, Liu B, et al. Dapagliflozin (DAPA) reduces HbA1c in Asian patients with T2DM after metformin (MET) failure [abstract]. Diabetes 2014;63(Suppl 1):A279.

15. Iijima H, Kifuji T, Maruyama N, et al. Pharmacokinetics, pharmacodynamics, and safety of canagliflozin in Japanese patients with type 2 diabetes mellitus. Adv Ther 2015;32:768-82.

16. Devineni D, Polidori D. Clinical pharmacokinetic, pharmacodynamic, and drug-drug interaction profile of canagliflozin, a sodium-glucose co-transporter 2 inhibitor. Clin Pharmacokinet 2015;54:1027-41.

17. Devineni D, Curtin CR, Polidori D, et al. Pharmacokinetics and pharmacodynamics of canagliflozin, a sodium glucose co-transporter 2 inhibitor, in subjects with type 2 diabetes mellitus. $\mathcal{F}$ Clin Pharmacol 2013;53:601-10.

18. Kapur A, O'Connor-Semmes R, Hussey EK, et al. First human dose-escalation study with remogliflozin etabonate, a selective inhibitor of the sodium-glucose transporter 2 (SGLT2), in healthy subjects and in subjects with type 2 diabetes mellitus. BMC Pharmacol Toxicol 2013;14:26.

19. Dobbins RL, O'Connor-Semmes R, Kapur A, et al. Remogliflozin etabonate, a selective inhibitor of the sodium-dependent transporter 2 reduces serum glucose in type 2 diabetes mellitus patients. Diabetes Obes Metab 2012;14: $15-22$.

20. Veltkamp SA, Kadokura T, Krauwinkel WJJ, et al. Effect of ipragliflozin (ASP1941), a novel selective sodium-dependent glucose co-transporter 2 inhibitor, on urinary glucose excretion in healthy subjects. Clin Drug Investig 2011;31:839-51.

21. Kadokura T, Akiyama N, Kashiwagi A, et al. Pharmacokinetic and pharmacodynamic study of ipragliflozin in Japanese patients with type 2 diabetes mellitus: a randomized, double-blind, placebo-controlled study. Diabetes Res Clin Pract 2014;106:50-6.

22. Amin NB, Wang X, Mitchell JR, et al. Blood pressure-lowering effect of the sodium glucose co-transporter-2 inhibitor ertugliflozin, assessed via ambulatory blood pressure monitoring in patients with type 2 diabetes and hypertension. Diabetes Obes Metab 2015;17:805-8.

23. Ikeda S, Takano Y, Cynshi O, et al. A novel and selective sodium-glucose cotransporter-2 inhibitor, tofogliflozin, improves glycaemic control and lowers body weight in patients with type 2 diabetes mellitus. Diabetes Obes Metab 2015;17:984-93.

24. Zambrowicz B, Lapuerta P, Strumph P, et al. LX4211 therapy reduces postprandial glucose levels in patients with type 2 diabetes mellitus and renal impairment despite low urinary glucose excretion. Clin Ther 2015;37:71-82.e12.

25. Rosenstock J, Cefalu W'T, Lapuerta P, et al. Greater dose-ranging effects on A1C levels than on glucosuria with LX4211, a dual inhibitor of SGLT1 and SGLT2, in patients with type 2 diabetes on metformin monotherapy. Diabetes Care 2015;38:431-8.

26. Hutton B, Salanti G, Caldwell DM, et al. The PRISMA extension statement for reporting of systematic reviews incorporating network meta-analyses of health care interventions: checklist and explanations. Ann Intern Med 2015; $162: 777-84$ 
27. Higgins JPT, Green S, editors. Cochrane handbook for systematic reviews of interventions. Version 5.1.0. Oxford (UK): Cochrane Collaboration; 2011. Available: http://handbook-5-1.cochrane.org/ (accessed 2016 May 1).

28. Khangura S, Konnyu K, Cushman R, et al. Evidence summaries: the evolution of a rapid review approach. Syst Rev 2012;1:10.

29. Henry RR, Murray AV, Marmolejo MH, et al. Dapagliflozin, metformin XR, or both: initial pharmacotherapy for type 2 diabetes, a randomised controlled trial. Int 7 Clin Pract 2012;66:446-56.

30. Tanizawa Y, Kaku K, Araki E, et al.; Tofogliflozin 004 and 005 Study Group. Long-term safety and efficacy of tofogliflozin, a selective inhibitor of sodiumglucose cotransporter 2 , as monotherapy or in combination with other oral antidiabetic agents in Japanese patients with type 2 diabetes mellitus: multicenter, open-label, randomized controlled trials. Expert Opin Pharmacother 2014;15:749-66.

31. Ferrannini E, Berk A, Hantel S, et al. Long-term safety and efficacy of empagliflozin, sitagliptin, and metformin: an active-controlled, parallel-group, randomized, 78-week open-label extension study in patients with type 2 diabetes. Diabetes Care 2013;36:4015-21.

32. Liu XY, Zhang N, Chen R, et al. Efficacy and safety of sodiumglucose cotransporter 2 inhibitors in type 2 diabetes: a meta-analysis of randomized controlled trials for 1 to 2 years. 7 Diabetes Complications 2015;29: 1295-303.

33. Zaccardi F, Webb DR, Htike ZZ, et al. Efficacy and safety of sodium-glucose co-transporter-2 inhibitors in type 2 diabetes mellitus: systematic review and network meta-analysis. Diabetes Obes Metab 2016;18:783-94.

34. Vasilakou D, Karagiannis T, Athanasiadou E, et al. Sodium-glucose cotransporter 2 inhibitors for type 2 diabetes: a systematic review and meta-analysis. Ann Intern Med 2013;159:262-74.

35. Berhan A, Berhan Y. Efficacy of alogliptin in type 2 diabetes treatment: a meta-analysis of randomized double-blind controlled studies. BMC Endocr Disord 2013;13:9

36. Wu JH, Foote C, Blomster J, et al. Effects of sodium-glucose cotransporter-2 inhibitors on cardiovascular events, death, and major safety outcomes in adults with type 2 diabetes: a systematic review and meta-analysis. Lancet Diabetes Endocrinol 2016;4:411-9.

37. Kawalec P, Mikrut A, Łopuch S. The safety of dipeptidyl peptidase-4 (DPP4) inhibitors or sodium-glucose cotransporter 2 (SGLT-2) inhibitors added to metformin background therapy in patients with type 2 diabetes mellitus: a systematic review and meta-analysis. Diabetes Metab Res Rev 2014;30:269-83.

38. Puckrin R, Saltiel MP, Reynier P, et al. SGLT-2 inhibitors and the risk of infections: a systematic review and meta-analysis of randomized controlled trials. Acta Diabetol 2018;55:503-14.
39. Abu-Ashour W, Twells L, Valcour J, et al. The association between diabetes mellitus and incident infections: a systematic review and meta-analysis of observational studies. BM7 Open Diabetes Res Care 2017;5:e00336.

40. Chen SL, Jackson SL, Boyko EJ. Diabetes mellitus and urinary tract infection: epidemiology, pathogenesis and proposed studies in animal models. 7 Urol 2009;182(Suppl):S51-6.

41. Daneshgari F, Liu G, Birder L, et al. Diabetic bladder dysfunction: current translational knowledge. 7 Urol 2009;182(Suppl):S18-26.

42. Geerlings SE, Meiland R, van Lith EC, et al. Adherence of type 1-fimbriated Escherichia coli to uroepithelial cells: more in diabetic women than in control subjects. Diabetes Care 2002;25:1405-9.

Affiliations: School of Pharmacy (Donnan, Grandy, Chibrikov, Marra, Johnston, Hache, Curnew, Nguyen, Gamble) and Faculty of Medicine (Aubrey-Bassler, Swab), Memorial University, St. John's, NL; School of Pharmacy (Marra), University of Otago, Dunedin, New Zealand; School of Pharmacy (Gamble), University of Waterloo, Kitchener, Ont.

Contributors: Jennifer Donnan led the review and was involved at every stage, including protocol development, search strategy design, screening, data extraction, quality appraisal, analysis and manuscript preparation. Catherine Grandy was involved in screening, data extraction, quality appraisal and manuscript revisions. Eugene Chibrikov was involved in data cleaning and analysis, and manuscript revisions. Carlo Marra, Kris Aubrey-Bassler and Karissa Johnston were involved in project conception, protocol development and manuscript revisions. Michelle Swab was involved in search strategy design, literature search and manuscript revisions. Jenna Hache and Daniel Curnew were involved in screening, data extraction, quality appraisal and manuscript revisions. Hai Nguyen was involved in interpretation of study results and manuscript revisions. JohnMichael Gamble supervised this research and was involved in protocol development, consensus on disagreements in data extraction, data analysis, interpretation of results and manuscript revisions. All of the authors gave approval of the version to be published and agreed to be accountable for all aspects of the work

Supplemental information: For reviewer comments and the original submission of this manuscript, please see www.cmajopen.ca/content/6/4/ E594/suppl/DC1. 